\title{
Insights into COVID-19 Chemotherapies: Potential and Challenges
}

\section{Esam Bashir Yahya *}

School of Industrial Technology, Universiti Sains Malaysia, Penang 11800, Malaysia

\begin{tabular}{lllllll}
\hline Received: 08.02.2021 & • & Accepted: 15.03.2021 & • & Published: 30.04.2021 & • & Final Version: 30.04.2021 \\
\hline
\end{tabular}

\begin{abstract}
The coronavirus disease 2019 (COVID-19), a newly emerged infectious disease caused by SARS-CoV-2 virus, has recently become pandemic. Although several therapeutic options are currently available for the treatment of COVID-19, no effective antiviral agents have been developed yet. Many countries follow the strategy of keeping the patient in good state and count on his own immune system to develop an effective immune response. Since the beginning of the pandemic, many previous therapeutic options have been used in COVID-19 treatment including antiviral, nonantiviral drugs and convalescent plasma-based therapies. This review delivers comprehensive illustration of the current therapeutic drugs that have been used for COVID-19 treatment all over the world since the pandemic starts.
\end{abstract}

Keywords: COVID-19 infection, therapeutic drug, antiviral drug, non-antiviral drug.

\section{Introduction}

Severe acute respiratory syndrome coronavirus II (SARS-CoV-2), commonly known as COVID-19, was identified for the first time in December 2019 in China as the cause of a novel respiratory illness designated coronavirus disease (Helmy et al., 2020). Since then, several drugs and therapeutic options have been evaluated for the treatment of COVID-19, including traditional alternative medicine previously available antiviral drugs and novel drugs. However, no antiviral agents were found to be fully effective against this viral disease. Most agents only enhance or lower the acute phase of the disease (Borba et al., 2020; B. Cao et al., 2020). COVID-19 is a threat to millions globally, as immunity does not exist and more than $20 \%$ of patients develop severe disease. The virus has the capacity to escape the innate immune responses through various mechanisms, which increase its pathogenicity and proliferation in primarily infected tissues. Many attempts have been initiated since the beginning of the pandemic by repurposing broad-spectrum antivirals drugs for the immediate treatment COVID-19. Favipiravir, remdesivir, lopinavir, nitazoxanide, ivermectin and ribavirin, etc., are the most used antiviral drugs that entered significant number of clinical trials. The antiviral activity of these drugs are known, as most of them have been successfully used to treat previous viral diseases such as influenza, Ebola, HIV, MERS Cov and SARS Cov (X. Cao, 2020). Other non-antiviral therapeutics include antimalarial drugs such as chloroquine and hydroxychloroquine; immune regulators complexes such as corticosteroids and interferons in addition to the use of polyclonal and monoclonal antibodies (Boulware et al., 2020). These therapies have raised the survival rate among COVID-19 patients and significantly lowered mortality rate.

\footnotetext{
* Corresponding Author: essam912013@gmail.com
} 
This review present comprehensive summary of all the therapeutic drugs that have been used in COVID-19 treatment all over the world.

\section{COVID-19 immunology}

Recent studies revealed that approximately $80 \%$ of COVID-19 infections are mild or asymptomatic, while the remaining $20 \%$ of infections are tend to be severe or critical unwell (Chan et al., 2020; C. Huang et al., 2020). The spike proteins of COVID-19 tightly bind to the host cell receptor, as it considers 20-30 amino acids longer than the previous Middle East Respiratory Syndrome-Related Coronavirus (MERS-CoV) and Severe Acute Respiratory Syndrome-Related Coronavirus (SARS$\mathrm{CoV}$ ) and other closely related coronaviruses (Prompetchara, Ketloy, \& Palaga, 2020). Coronaviruses including COVID-19 use ACE2 receptor as their host cell receptor to initiate the infection (Zhou et al., 2020). This receptor is expressed almost in all of our cells and organs, and it has been proved to over expressed on the upper respiratory tract, which provides a portal of entry for the virus in humans (Sungnak, Huang, Bécavin, Berg, \& Network, 2020). Infection of various immune cells including macrophages, monocytes and $\mathrm{T}$ cells has been observed in COVID-19 infections, the exact mechanism and to what extent COVID-19 can infect these cell types is not clear to date. These immune cells express ACE2, but at lower levels and not ubiquitously. This may provide an entry mechanism for COVID-19 to them. However, other receptors and immune complexes may be involved in entry mechanism (Zhu et al., 2020). Figure 1 presents ACE2 shedding hypothesis of COVID-19 entry.

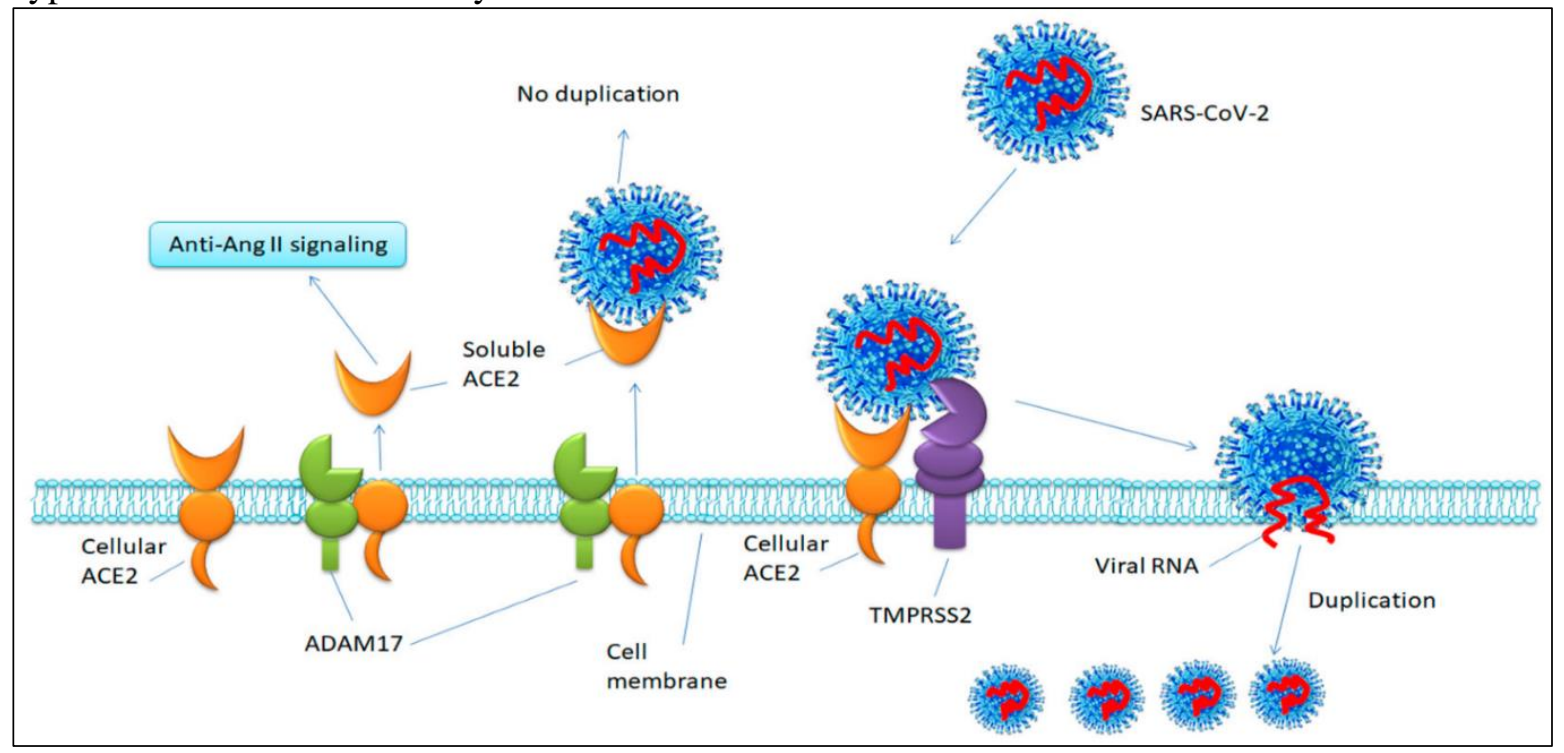

Figure 1. Schematic illustration of ACE2 shedding hypothesis of COVID-19 entry. Adapted from (Xiao, Sakagami, \& Miwa, 2020)

After the entry of COVID-19 in the host cells, immediate viral response is occurred through the release of type I interferon and down-stream signals to modulate anti-viral cell responses (Addi et al., 2008). The immune cells then identify the viral RNA and other viral derived pattern associated molecular patterns and bind to them for pattern recognition receptors activation in/on immune cells leading to their activation for the viral response. Different toll-like receptors on the immune cells can recognize the viral RNA, which leads to nuclear translocation of many transcription factors (such as NFKB and IRF3). It also leads to the increase of the expression of type I interferon as well as other innate pro-inflammatory cytokines including IL-1, IL-6, TNF- $\alpha$ and NFKB (Prompetchara et al., 
2020). The release of these immune complexes promote the expression of other immune complexes: interferon I activate the IFN- $\alpha$ receptor complex, which will results in the activation and/or phosphorylation of signal transducers and activators of transcription (STAT family) transcription factors 1 and 2. However, the activation of IL-1, 6, and TNF receptor feeds into the expression of pro-inflammatory cytokine via the transcription factor NFאB (Alunno, Padjen, Fanouriakis, \& Boumpas, 2019). COVID-19 can counteract type I interferon signaling through the inhibition of the phosphorylation of signal transducers and activators of transcription factor family, leading to stop the antiviral response of the host cells (Yi, Lagniton, Ye, Li, \& Xu, 2020). The suppression of host innates immune mechanisms in the infected epithelial cells and/or infected immune cells allow COVID-19 to proliferate without triggering the host innate anti-viral response machinery. COVID19 may also escape from the host immune system through apoptosis induction to the T cell, and thus give the virus more time for replication and invasion (Yi et al., 2020). However, lymphocytes ( $\mathrm{T}$ and B cells) may also become depleted, as a results of over expression of pro-inflammatory cytokines, which is known as cytokine storm (Soy et al., 2020).

\section{Antiviral based therapeutic drugs for Covid-19}

Most of COVID-19 patients have been reported with mild or moderate courses. Only 5\%-10\% showed severe or even potentially life-threatening course (YAVUZ \& Ünal, 2020). Significant numbers of antiviral and immune modulating therapeutics have been evaluated in various stages of treatment evaluation, many of them already reached clinical trials and some of them maybe published in the next few months. This review presents the most frequently used antiviral drugs all over the world.

\subsection{Remdesivir}

Remdesivir is an antiviral drug, which can stop viral replication by inhibiting viral RNA-dependent, RNA polymerase. It has been successfully used against previous similar viruses including Middle East Respiratory Syndrome (MERS-CoV) and show effective results (Sheahan et al., 2017; Sheahan et al., 2020). Remdesivir was identified and entered the clinical trials as a promising therapeutic drug since the early months of COVID-19 pandemic due to its in vitro ability to inhibit COVID-19 replication (M. Wang et al., 2020). Madsen et al. (Madsen, 2020) evaluated the effectiveness of remdesivir compared to placebo drug on 1062 COVID-19 patients. The authors reported that patients who received remdesivir show median recovery time of 10 days with significant clinical improvement compared with those received the placebo 15 days. Wang et al. (M. Wang et al., 2020) in vitro evaluated remdesivir antiviral activities against COVID-19 and revealed its ability to block the viral infection even at low concentrations with minimal cytotoxicity. However, remdesivir was interrupted in four COVID-19 patients before initial planned duration, significant elevation in alanine aminotransferase was observed in two of them and renal failure requiring renal replacement in the other two (Dubert et al., 2020). Similarly, Wang et al. (Y. Wang et al., 2020) reported no significant clinical benefit in patient who received remdesivir for all the planed duration of time. Grein et al. (Grein et al., 2020) described the common adverse events of using remdesivir in COVID19 treatment, they reported rash, hypotension, diarrhea, renal impairment and abnormal liver function. In addition to serious adverse events in some patients including acute kidney injury, multiorgan failure and septic shock. The safety profile of remdesivir for COVID-19 treatment is yet incompletely characterized, data from previous studies suggest no specific alarm or stop using remdesivir for COVID-19 treatment. Remdesivir should be used under a proper pharmacovigilance 
for COVID-19 treatment, with paying specific attention for disproportionate rise in alanine transaminase or decrease in glomerular filtration rate during the treatment with remdesivir.

\subsection{Favipiravir}

Favipiravir is an antiviral agent that inhibits the viral RNA-dependent RNA polymerase. This drug must transform to its active form inside the cell by undergoing intracellular phosphoribosylation, the resulted active form then recognized as a substrate by RNA-dependent RNA polymerase, and inhibits the viral RNA polymerase activity (Coomes \& Haghbayan, 2020). Favipiravir designed first time to treat influenza in addition to trialled for other diseases such as Ebola. Shenzhen et al. (C. Chen et al., 2020) conducted observational study and showed a significantly faster viral clearance in patient who used favipiravir compared to other drugs such as lopinavir/ritonavir. In different study, Pilkington et al. (Pilkington, Pepperrell, \& Hill, 2020) reviewed twenty-nine studies and a total of 4299 participants assessing using favipiravir to treat COVID-19 infection by 27 March 2020 and revealed significantly fewer gastrointestinal adverse events occurring on favipiravir with significant elevation in uric acid compared to other drugs such as umifenovir, oseltamivir, lopinavir and ritonavir. Cai et al. (Cai et al., 2020) reported shorter viral clearance time and significant improvement in chest $\mathrm{CT}$ in patients who used favipiravir compared with the control. The same authors reported fewer adverse events to favipiravir based treatment and better therapeutic responses on COVID-19 infection in terms of disease progression as well as viral clearance. Despite being the favorable option for many medical professionals and its low adverse events. However, safety concerns remain with the use of Favipiravir for COVID-19 treatment: hyperuricaemia, QTc prolongation and teratogenicity are remain serious challenges and need to be adequately studied. Most of studies evaluated the adverse events of Favipiravir in short-term use, which showed safe and tolerable, assessing the longer-term effects of treatment is needed to ensure the complete safety of such treatment.

\subsection{Lopinavir/ritonavir}

Lopinavir is an antiviral drug work by inhibiting protease enzyme, which is necessary for viral infection, and it is normally used with ritonavir as a booster. Protease is a key enzyme for COVID19 and most of coronavirus polyprotein processing and thus, these drugs have been proposed for being potential treatment for COVID-19 as they possess strong in vitro anti coronavirus activity (Kang et al., 2020). Park et al. (Park et al., 2020) used lopinavir-ritonavir to treat COVID-19 animals and reported significant reduce in clinical symptoms but the virus titres remain the same. A previous study on using lopinavir-ritonavir to treat 199 COVID-19 patients showed no significant improvement in duration of hospital stay, viral load, or mortality (B. Cao et al., 2020). Horby et al. (Horby et al., 2020) evaluated the potential use of lopinavir-ritonavir based treatment and observed no significant difference in term of discharging time and the proportion of patients who survived after invasive mechanical ventilation or died.

\subsection{Nitazoxanide}

Nitazoxanide display broad-spectrum antiviral properties and showed promising pharmacodynamics against COVID-19. It is a pro-drug for tizoxanide and has many viral indications (Srivatsan Padmanabhan, 2020). The antiviral activity of Nitazoxanide resulted from interfering with host-regulated pathways, which involved in viral replication rather than virus specific pathways. Thus, it was nominated as a good candidate for potentially inhibiting COVID-19. The in vitro 
antiviral efficacy of nitazoxanide found promising against COVID-19 compared with other antiviral drug such as remdesivir, antimalarial chloroquine and superior to favipiravir (M. Wang et al., 2020). Number of clinical trials have been conducted using nitazoxanide, Kelleni has suggested using new protocol for early cases of COVID-19, which consist of nitazoxanide with azithromycin (Kelleni, 2020). In different study, Pepperrell et al. (Pepperrell, Pilkington, Owen, Wang, \& Hill, 2020) reviewed the clinical studies about nitazoxanide to determine its safety and they calculated the minimum production cost of the drug for the expected anti-viral potential use in COVID-19 treatment. There are some challenges of using nitazoxanide in COVID-19 treatment, some safety issues especially in children and during pregnancy, the number of clinical studies of using nitazoxanide in COVID-19 treatment is still not sufficient.

\subsection{Ivermectin}

Ivermectin is a broad-spectrum antiparasitic drug with demonstrated strong antiviral activity against both DNA and RNA viruses, including COVID-19 (Formiga et al., 2020). Caly et al. (Caly, Druce, Catton, Jans, \& Wagstaff, 2020) demonstrated that a single dose of ivermectin reduced the replication of COVID-19 virus in Vero/hSLAM cells by 5000 -fold. Ivermectin said to be act by preventing the viral proteins from entering the cells, and thus prevent the replication of the virus (Caly et al., 2020). Alam et al. (Alam et al., 2020) conducted observational study and revealed that single-dose of ivermectin yielded considerable improvements in COVID-19 symptoms and the viral response. A recent retrospective study done by Rajter et al. (Rajter et al., 2020) found that hospitalized COVID-19 patients given ivermectin drug with other treatments had significantly lower mortality rates compared with the patients who did not receive ivermectin.

\subsection{Ribavirin}

A guanosine analogue generalized antiviral drug have been used to several types of viral infections including respiratory syncytial virus, hepatitis $C$ virus and other viral hemorrhagic fevers (Chan et al., 2015). With the previous experience of using ribavirin to treat Middle East Respiratory Syndrome in 2012 and rationales, it has been put into clinical trials in COVID-19 and showed variable and undesirable adverse effect in some patients of reducing hemoglobin (Martinez, 2020). A randomized, open-label clinical trial done by Huang et al. (Y.-Q. Huang et al., 2020) in China evaluated the effectiveness of combination of ribavirin and interferon- $\alpha$ in patients with mild to moderate COVID19. The authors reported no significant differences in terms of antiviral effectiveness of ribavirin. In recent study evaluated the effect of ribavirin/ IFN- $\alpha$ COVID-19 therapy, the authors revealed no benefit in improving clinical outcomes, suggesting that ribavirin/ IFN- $\alpha$ should be avoided in COVID-19 patients (Li et al., 2021).

\section{Non-Antiviral based therapeutic drugs for COVID-19}

Several non-viral based therapeutic agents have been developed and used for COVID-19 treatment including the famous antimalarial drugs chloroquine and hydroxychloroquine, which showed interestingly strong effect against the novel coronavirus. Many types of corticosteroids have been also used and entered the clinical trials with variable results. However, using convalescent plasma transfusion and the specific antibodies have showed the safest and the most effective results in most of cases as we present down in this review. 


\subsection{Antimalarial and autoimmune disease drug}

Chloroquine and hydroxychloroquine are two aminoquinolines have been used for over 50 years to treat malaria as well as some autoimmune diseases (Colson, Rolain, Lagier, Brouqui, \& Raoult, 2020). These two drugs possess immunomodulatory effects beside their antimalarial effects, allowing their usage in some autoimmune conditions such as rheumatoid arthritis and systemic lupus erythematosus (Jakhar \& Kaur, 2020). Their ability to inhibit certain cellular functions in addition to molecular pathways involved in immune activation by their accumulation in the lysosomes and auto phagosomes of macrophages and other phagocytic cells, which change the local pH concentrations. As a weak diprotic bases, chloroquine tends to concentrate within acidic organelles within the cell such as lysosomes and endosomes, leading to elevate the intra-vesicular $\mathrm{pH}$ and preventing of endosome trafficking as well as viral fusion into the cell (Schrezenmeier \& Dörner, 2020). This mechanism of these drugs has translated to promising role of Chloroquine and hydroxychloroquine in the treatment of COVID-19, some recent studies revealed that they also interferes with ACE-2 receptor glycosylation, which prevents COVID-19 receptor binding and subsequent infection (Funck-Brentano \& Salem, 2020). Kashour et al. (Kashour et al., 2021) systematically evaluated the effect of chloroquine and hydroxychloroquine for COVID-19 patients. The authors concluded that moderate certainty evidence suggests that hydroxychloroquine lacks efficacy in reducing COVID-19 patient short-term mortality or risk of hospitalization in outpatients. Other clinical trials on chloroquine have been done on COVID-19 Chinese patients, showed a great effect of the drug in terms of clinical results as well as viral clearance (Gautret et al., 2020).

\subsection{Corticosteroids}

Many corticosteroids have been used for the treatment of previous coronaviruses as well as the novel coronavirus of COVID-19. Viral particles can stimulate our innate immune response, which activates the complement system and alveolar macrophages. As a result of these activations, other consecutive activations are occurred leading to massive inflammatory response, which cause microvascular thromboses and alveolar and vascular damage (Ciceri et al., 2020; Tan et al., 2020). In the late stages of COVID-19 disease, generalized systemic inflammatory reaction have been reported, which involve other organs and cause multi-organ failure and ending up with death. Theoretically, corticosteroid-based treatment act as immune response suppressor, which prevent the lung inflammation and pathogen clearance. A recent clinical trial conducted by RECOVERY Collaborative Group (Group, 2020) showed that the use of dexamethasone (type of corticosteroid) resulted in lower 28-day mortality in COVID-19 patients. The updated living WHO guideline in 2020 on COVID-19 therapeutic drugs suggests not to use any of the corticosteroids in non-severe COVID-19 treatment but with a weak or conditional recommendation (Lamontagne et al., 2020). Pasin et al. (Pasin et al., 2021) evaluated the treatment of COVID-19 using corticosteroids and concluded that they may only be considered in the severe critically cases of COVID-19. The authors recommend that corticosteroids must be discouraged in COVID-19 patients who does not requiring oxygen therapy. Chen et al. (Q. Chen et al., 2021) reported that using corticosteroid therapy for treatment of severe and critical COVID-19 patients leaded to significant lower of mortality rate, but with induction of variable side effects. In different study, Yuan et al. [38] reported that using corticosteroid in COVID-19 treatment might harm lung injury recovery especially in non-severe pneumonia patients. The use of corticosteroid in COVID-19 treatment is restricted due to some health concerns associated with this drug, and it should be always used carefully. 


\subsection{Convalescent plasma transfusion}

Convalescent plasma refers to the collected plasma from recovered patients, which contain specific antibodies of the disease. Convalescent plasma therapy has been used in to significantly improve the survival rate of COVID-19 patients as well as other viral infections (L. Chen, Xiong, Bao, \& Shi, 2020; Shen et al., 2020). Plasma may be obtained either from the whole blood of recovered COVID19 survivors or via apheresis. Yields in apheresis collection are more units of convalescent plasma and thus this method is strongly preferred than standard blood donation (Henney \& Shalala, 1997). Many studies reported the successful treatment of individuals with severe COVID-19, which demonstrates the potential efficacy of convalescent plasma therapy (Duan et al., 2020; Zhang et al., 2020).

The immunoglobulin antibodies within the plasma of COVID-19 recovered patients proved to suppress viremia. The specific antibodies can act as prophylactic and therapeutic tools that target specific viral spike proteins and/or bind to hemagglutinin in viral infections. Numerous studies have shown that COVID-19 spike protein play vital role in mediating viral entry into the host cells (Martinez, 2020). Shen et al. (Shen et al., 2020) reported on five COVID-19 critically ill patients who received plasma transfusion containing a COVID-19-specific antibody obtained from recovered patients. The authors observed improvements in clinical condition and decline in viral loads following plasma transfusions, concluding that the use of convalescent plasma therapy is strongly beneficial among COVID-19 infected patients. Alsharidah et al. (Alsharidah et al., 2021) evaluated the effectiveness of convalescent plasma collected from recovered COVID-19 patients in treatment of other moderate and severe COVID-19 disease. The authors concluded based on their finding that administration of convalescent plasma is a safe treatment option for both moderate and severe COVID-19 patients.

\section{Conclusion}

Many attempts have been initiated since the beginning of the pandemic by repurposing broadspectrum antivirals drugs for the immediate treatment COVID-19. Although many of them have successfully lower the rate of mortality and shorted the treatment time, still there is no effective antiviral agents have been developed yet. This review delivered comprehensive illustration of the current therapeutic drugs that have been used for COVID-19 treatment all over the world since the pandemic starts. 


\section{References}

[1] Addi, A. B., Lefort, A., Hua, X., Libert, F., Communi, D., Ledent, C., . . Robaye, B. (2008). Modulation of murine dendritic cell function by adenine nucleotides and adenosine: involvement of the A2B receptor. European journal of immunology, 38(6), 1610-1620.

[2] Alam, M. T., Murshed, R., Bhiuyan, E., Saber, S., Alam, R. F., \& Robin, R. C. (2020). A case series of 100 COVID-19 positive patients treated with combination of ivermectin and doxycycline. Journal of Bangladesh College of Physicians and Surgeons, 10-15.

[3] Alsharidah, S., Ayed, M., Ameen, R. M., Alhuraish, F., Rouheldeen, N. A., Alshammari, F. R., ... Dawoud, M. E. (2021). COVID-19 convalescent plasma treatment of moderate and severe cases of SARS-CoV-2 infection: A multicenter interventional study. International Journal of Infectious Diseases, 103, 439-446.

[4] Alunno, A., Padjen, I., Fanouriakis, A., \& Boumpas, D. T. (2019). Pathogenic and therapeutic relevance of JAK/STAT signaling in systemic lupus erythematosus: integration of distinct inflammatory pathways and the prospect of their inhibition with an oral agent. Cells, 8(8), 898.

[5] Borba, M. G. S., Val, F. F. A., Sampaio, V. S., Alexandre, M. A. A., Melo, G. C., Brito, M., . . Guerra, M. V. F. (2020). Effect of high vs low doses of chloroquine diphosphate as adjunctive therapy for patients hospitalized with severe acute respiratory syndrome coronavirus 2 (SARS-CoV-2) infection: a randomized clinical trial. JAMA network open, 3(4), e208857-e208857.

[6] Boulware, D. R., Pullen, M. F., Bangdiwala, A. S., Pastick, K. A., Lofgren, S. M., Okafor, E. C., ... Abassi, M. (2020). A randomized trial of hydroxychloroquine as postexposure prophylaxis for Covid-19. New England Journal of Medicine, 383(6), 517-525.

[7] Cai, Q., Yang, M., Liu, D., Chen, J., Shu, D., Xia, J., . . Yang, Y. (2020). Experimental treatment with favipiravir for COVID-19: an open-label control study. Engineering, 6(10), 1192-1198.

[8] Caly, L., Druce, J. D., Catton, M. G., Jans, D. A., \& Wagstaff, K. M. (2020). The FDA-approved drug ivermectin inhibits the replication of SARS-CoV-2 in vitro. Antiviral research, 178, 104787.

[9] Cao, B., Wang, Y., Wen, D., Liu, W., Wang, J., Fan, G., . . Wei, M. (2020). A trial of lopinavir-ritonavir in adults hospitalized with severe Covid-19. New England Journal of Medicine.

[10] Cao, X. (2020). COVID-19: immunopathology and its implications for therapy. Nature reviews immunology, 20(5), 269-270.

[11] Chan, J. F.-W., Yao, Y., Yeung, M.-L., Deng, W., Bao, L., Jia, L., . . . Yu, P. (2015). Treatment with lopinavir/ritonavir or interferon- $\beta 1 \mathrm{~b}$ improves outcome of MERS-CoV infection in a nonhuman primate model of common marmoset. The Journal of infectious diseases, 212(12), 1904-1913.

[12] Chan, J. F.-W., Yuan, S., Kok, K.-H., To, K. K.-W., Chu, H., Yang, J., . . Poon, R. W.-S. (2020). A familial cluster of pneumonia associated with the 2019 novel coronavirus indicating person-to-person transmission: a study of a family cluster. The Lancet, 395(10223), 514-523.

[13] Chen, C., Huang, J., Cheng, Z., Wu, J., Chen, S., Zhang, Y., . . Zhang, J. (2020). Favipiravir versus arbidol for COVID-19: a randomized clinical trial. MedRxiv.

[14] Chen, L., Xiong, J., Bao, L., \& Shi, Y. (2020). Convalescent plasma as a potential therapy for COVID-19. The Lancet infectious diseases, 20(4), 398-400. 
[15] Chen, Q., Song, Y., Wang, L., Zhang, Y., Han, L., Liu, J., . . Wang, T. (2021). Corticosteroids treatment in severe patients with COVID-19: a propensity score matching study. Expert review of respiratory medicine.

[16] Ciceri, F., Beretta, L., Scandroglio, A. M., Colombo, S., Landoni, G., Ruggeri, A., . . Rovere-Querini, P. (2020). Microvascular COVID-19 lung vessels obstructive thromboinflammatory syndrome (MicroCLOTS): an atypical acute respiratory distress syndrome working hypothesis. Critical care and resuscitation, 22(2), 95.

[17] Colson, P., Rolain, J.-M., Lagier, J.-C., Brouqui, P., \& Raoult, D. (2020). Chloroquine and hydroxychloroquine as available weapons to fight COVID-19. Int J Antimicrob Agents, 55(4), 105932.

[18] Coomes, E. A., \& Haghbayan, H. (2020). Favipiravir, an antiviral for COVID-19? Journal of Antimicrobial Chemotherapy, 75(7), 2013-2014.

[19] Duan, K., Liu, B., Li, C., Zhang, H., Yu, T., Qu, J., . . Hu, Y. (2020). Effectiveness of convalescent plasma therapy in severe COVID-19 patients. Proceedings of the National Academy of Sciences, 117(17), 94909496.

[20] Dubert, M., Visseaux, B., Isernia, V., Bouadma, L., Deconinck, L., Patrier, J., . . Rioux, C. (2020). Case report study of the first five COVID-19 patients treated with remdesivir in France. International Journal of Infectious Diseases, 98, 290-293.

[21] Formiga, F. R., Leblanc, R., de Souza Rebouças, J., Farias, L. P., de Oliveira, R. N., \& Pena, L. (2020). Ivermectin: an award-winning drug with expected antiviral activity against COVID-19. Journal of controlled release.

[22] Funck-Brentano, C., \& Salem, J.-E. (2020). RETRACTED: Chloroquine or hydroxychloroquine for COVID-19: why might they be hazardous? The Lancet.

[23] Gautret, P., Lagier, J.-C., Parola, P., Meddeb, L., Mailhe, M., Doudier, B., . . . Dupont, H. T. (2020). Hydroxychloroquine and azithromycin as a treatment of COVID-19: results of an open-label nonrandomized clinical trial. International journal of antimicrobial agents, 56(1), 105949.

[24] Grein, J., Ohmagari, N., Shin, D., Diaz, G., Asperges, E., Castagna, A., . . . Lescure, F.-X. (2020). Compassionate use of remdesivir for patients with severe Covid-19. New England Journal of Medicine, 382(24), 2327-2336.

[25] Group, R. C. (2020). Dexamethasone in hospitalized patients with Covid-19-preliminary report. New England Journal of Medicine.

[26] Helmy, Y. A., Fawzy, M., Elaswad, A., Sobieh, A., Kenney, S. P., \& Shehata, A. A. (2020). The COVID19 pandemic: a comprehensive review of taxonomy, genetics, epidemiology, diagnosis, treatment, and control. Journal of clinical medicine, 9(4), 1225.

[27] Henney, J. E., \& Shalala, D. E. (1997). PART 640-ADDITIONAL STANDARDS FOR HUMAN BLOOD AND BLOOD PRODUCTS. Safety, 3.

[28] Horby, P. W., Mafham, M., Bell, J. L., Linsell, L., Staplin, N., Emberson, J., . . . Prudon, B. (2020). Lopinavir-ritonavir in patients admitted to hospital with COVID-19 (RECOVERY): a randomised, controlled, open-label, platform trial. The Lancet, 396(10259), 1345-1352. 
[29] Huang, C., Wang, Y., Li, X., Ren, L., Zhao, J., Hu, Y., . . . Gu, X. (2020). Clinical features of patients infected with 2019 novel coronavirus in Wuhan, China. The Lancet, 395(10223), 497-506.

[30] Huang, Y.-Q., Tang, S.-Q., Xu, X.-L., Zeng, Y.-M., He, X.-Q., Li, Y., . . Zhang, L. (2020). No statistically apparent difference in antiviral effectiveness observed among ribavirin plus interferon-alpha, lopinavir/ritonavir plus interferon-alpha, and ribavirin plus lopinavir/ritonavir plus interferon-alpha in patients with mild to moderate coronavirus disease 2019: results of a randomized, open-labeled prospective study. Frontiers in Pharmacology, 11, 1071.

[31] Jakhar, D., \& Kaur, I. (2020). Potential of chloroquine and hydroxychloroquine to treat COVID-19 causes fears of shortages among people with systemic lupus erythematosus. Nature medicine, 26(5), 632-632.

[32] Kang, C. K., Seong, M.-W., Choi, S.-J., Kim, T. S., Choe, P. G., Song, S. H., . . Oh, M.-d. (2020). In vitro activity of lopinavir/ritonavir and hydroxychloroquine against severe acute respiratory syndrome coronavirus 2 at concentrations achievable by usual doses. The Korean journal of internal medicine, 35(4), 728.

[33] Kashour, Z., Riaz, M., Garbati, M. A., AlDosary, O., Tlayjeh, H., Gerberi, D., . . Tleyjeh, I. M. (2021). Efficacy of chloroquine or hydroxychloroquine in COVID-19 patients: a systematic review and metaanalysis. Journal of Antimicrobial Chemotherapy, 76(1), 30-42.

[34] Kelleni, M. T. (2020). Nitazoxanide/azithromycin combination for COVID-19: A suggested new protocol for early management. Pharmacological Research, 157, 104874.

[35] Lamontagne, F., Agoritsas, T., Macdonald, H., Leo, Y.-S., Diaz, J., Agarwal, A., . . Calfee, C. S. (2020). A living WHO guideline on drugs for covid-19. bmj, 370.

[36] Li, H., Xiong, N., Li, C., Gong, Y., Liu, L., Yang, H., . . . Wang, J. (2021). Efficacy of ribavirin and interferon- $\alpha$ therapy for hospitalized patients with COVID-19: a multicenter, retrospective cohort study. International Journal of Infectious Diseases.

[37] Madsen, L. W. (2020). Remdesivir for the Treatment of Covid-19-Final Report. The New England Journal of Medicine, 338(19), 1813-1826.

[38] Martinez, M. A. (2020). Compounds with therapeutic potential against novel respiratory 2019 coronavirus. Antimicrobial agents and chemotherapy, 64(5).

[39] Park, S.-J., Yu, K.-M., Kim, Y.-I., Kim, S.-M., Kim, E.-H., Kim, S.-G., . . Jang, S.-G. (2020). Antiviral efficacies of FDA-approved drugs against SARS-CoV-2 infection in ferrets. MBio, 11(3).

[40] Pasin, L., Navalesi, P., Zangrillo, A., Kuzovlev, A., Likhvantsev, V., Hajjar, L. A., . . Landoni, G. (2021). Corticosteroids for Patients With Coronavirus Disease 2019 (COVID-19) With Different Disease Severity: A Meta-Analysis of Randomized Clinical Trials. Journal of cardiothoracic and vascular anesthesia, 35(2), 578-584.

[41] Pepperrell, T., Pilkington, V., Owen, A., Wang, J., \& Hill, A. M. (2020). Review of safety and minimum pricing of nitazoxanide for potential treatment of COVID-19. Journal of virus eradication, 6(2), 52-60.

[42] Pilkington, V., Pepperrell, T., \& Hill, A. (2020). A review of the safety of favipiravir-a potential treatment in the COVID-19 pandemic? Journal of virus eradication, 6(2), 45-51.

[43] Prompetchara, E., Ketloy, C., \& Palaga, T. (2020). Immune responses in COVID-19 and potential vaccines: Lessons learned from SARS and MERS epidemic. Asian Pac J Allergy Immunol, 38(1), 1-9. 
[44] Rajter, J. C., Sherman, M. S., Fatteh, N., Vogel, F., Sacks, J., \& Rajter, J.-J. (2020). ICON (Ivermectin in COvid Nineteen) Study: use of ivermectin is associated with lower mortality in hospitalized patients with COVID-19. Available at SSRN 3631261.

[45] Schrezenmeier, E., \& Dörner, T. (2020). Mechanisms of action of hydroxychloroquine and chloroquine: implications for rheumatology. Nature Reviews Rheumatology, 16(3), 155-166.

[46] Sheahan, T. P., Sims, A. C., Graham, R. L., Menachery, V. D., Gralinski, L. E., Case, J. B., . . Trantcheva, I. (2017). Broad-spectrum antiviral GS-5734 inhibits both epidemic and zoonotic coronaviruses. Science Translational Medicine, 9(396).

[47] Sheahan, T. P., Sims, A. C., Leist, S. R., Schäfer, A., Won, J., Brown, A. J., . . Clarke, M. O. (2020). Comparative therapeutic efficacy of remdesivir and combination lopinavir, ritonavir, and interferon beta against MERS-CoV. Nature communications, 11(1), 1-14.

[48] Shen, C., Wang, Z., Zhao, F., Yang, Y., Li, J., Yuan, J., . . Xing, L. (2020). Treatment of 5 critically ill patients with COVID-19 with convalescent plasma. Jama, 323(16), 1582-1589.

[49] Soy, M., Keser, G., Atagündüz, P., Tabak, F., Atagündüz, I., \& Kayhan, S. (2020). Cytokine storm in COVID-19: pathogenesis and overview of anti-inflammatory agents used in treatment. Clinical rheumatology, 39, 2085-2094.

[50] Srivatsan Padmanabhan, M. (2020). Potential dual therapeutic approach against SARS-CoV-2/COVID-19 with Nitazoxanide and Hydroxychloroquine: DO-10.13140/RG. 2.2. 28124.74882.

[51] Sungnak, W., Huang, N., Bécavin, C., Berg, M., \& Network, H. L. B. (2020). SARS-CoV-2 entry genes are most highly expressed in nasal goblet and ciliated cells within human airways. ArXiv.

[52] Tan, C. W., Low, J. G. H., Wong, W. H., Chua, Y. Y., Goh, S. L., \& Ng, H. J. (2020). Critically ill COVID19 infected patients exhibit increased clot waveform analysis parameters consistent with hypercoagulability. American journal of hematology.

[53] Wang, M., Cao, R., Zhang, L., Yang, X., Liu, J., Xu, M., . . Xiao, G. (2020). Remdesivir and chloroquine effectively inhibit the recently emerged novel coronavirus (2019-nCoV) in vitro. Cell research, 30(3), 269271.

[54] Wang, Y., Zhang, D., Du, G., Du, R., Zhao, J., Jin, Y., . . Lu, Q. (2020). Remdesivir in adults with severe COVID-19: a randomised, double-blind, placebo-controlled, multicentre trial. The Lancet, 395(10236), 1569-1578.

[55] Xiao, L., Sakagami, H., \& Miwa, N. (2020). ACE2: the key molecule for understanding the pathophysiology of severe and critical conditions of COVID-19: demon or angel? Viruses, 12(5), 491.

[56] YAVUZ, S., \& Ünal, S. (2020). Antiviral treatment of COVID-19. Turkish journal of medical sciences, 50(SI-1), 611-619.

[57] Yi, Y., Lagniton, P. N., Ye, S., Li, E., \& Xu, R.-H. (2020). COVID-19: what has been learned and to be learned about the novel coronavirus disease. International journal of biological sciences, 16(10), 1753.

[58] Zhang, B., Liu, S., Tan, T., Huang, W., Dong, Y., Chen, L., . . . Zhang, X. (2020). Treatment with convalescent plasma for critically ill patients with severe acute respiratory syndrome coronavirus 2 infection. Chest, 158(1), e9-e13. 
[59] Zhou, P., Yang, X.-L., Wang, X.-G., Hu, B., Zhang, L., Zhang, W., . . Huang, C.-L. (2020). A pneumonia outbreak associated with a new coronavirus of probable bat origin. Nature, 579(7798), 270-273.

[60] Zhu, N., Zhang, D., Wang, W., Li, X., Yang, B., Song, J., . . Lu, R. (2020). A novel coronavirus from patients with pneumonia in China, 2019. New England Journal of Medicine. 